\title{
APRIL, BCMA and TACI proteins are abnormally expressed in non-small cell lung cancer
}

\author{
HENGLI DOU, ZHAOHUA YAN, MENG ZHANG and XIAOXIN XU \\ Department of Neurosurgery, The Fourth Hospital of Jinan, Jinan, Shandong 250031, P.R. China
}

Received June 16, 2015; Accepted August 12, 2016

DOI: $10.3892 / \mathrm{ol} .2016 .5095$

\begin{abstract}
Lung cancer is the leading cause of cancer-associated mortality. Non-small cell lung cancer (NSCLC) accounts for $>80 \%$ of lung cancers. The overall survival for NSCLC is dismal, with a 5 -year survival of $<5 \%$ for patients. Thus, identifying an effective biomarker for early diagnosis of lung cancer is the first essential step to reduce mortality. It has been recognized that certain inflammatory and immune responses are important in lung cancer development and prevention. The present study demonstrated that, in NSCLC, a proliferation-inducing ligand (APRIL), B-cell maturation antigen (BCMA)and transmembrane activator and CAML interactor (TACI) proteins are abnormally expressed by immunohistochemistry, reverse transcription-quantitative polymerase chain reaction and western blotting. In addition, the expression of APRIL, BCMA and TACI were observed to be involved in extracellular signal-regulated kinase (ERK)1/2 activation in A549 cells. Overall, the present study provides evidence that APRIL and its receptors, BCMA and TACI, may play roles in the biological processes of NSCLC tumors through the ERK1/2 signaling pathway.
\end{abstract}

\section{Introduction}

Lung cancer is a major cause of cancer-associated mortality worldwide. Non-small cell lung cancer (NSCLC) accounts for $>80 \%$ of lung cancers. The overall survival for NSCLC is dismal, with a 5-year survival of $<5 \%$ for patients (1). Tumor necrosis factor (TNF) $\alpha$ has been detected in NSCLC (2-8); however, other members of this superfamily have not yet been studied in this malignancy, including a proliferation-inducing ligand (APRIL), B-cell maturation antigen (BCMA) and transmembrane activator and CAML interactor (TACI). APRIL, also known as TNF superfamily member 13, is a member of the TNF family, and is processed intracellularly

Correspondence to: $\mathrm{Dr}$ Xiaoxin Xu, Department of Neurosurgery, The Fourth Hospital of Jinan, 50 Shifan Road, Jinan, Shandong 250031, P.R. China

E-mail: xuxiaoxin_jn@126.com

Key words: APRIL, BCMA, TACI, lung cancer, ERK1/2 within the Golgi apparatus by furin pro-protein convertase prior to secretion (9). It binds to and BCMA, two members of the TNF receptor family. APRIL has been observed to be highly expressed in several tumor tissues, where it stimulates the growth of solid tumor cells (9) and promotes their development and progression $(10,11)$. Whether APRIL and its receptors BCMA and TACI serve roles in development of NSCLC is still not known.

The present study provides for the first time evidence of the overexpression of APRIL and its receptors BCMA and TACI in NSCLC tumors. In addition, it revealed that APRIL activates the mitogen-activated protein kinase (MAPK) extracellular signal-regulated kinase (ERK)1/2 in A549 cells. Our data suggest a possible new role of APRIL and its receptors BCMA and TACI in the regulation of lung cancer.

\section{Materials and methods}

Tumor samples. A total of 38 specimens of NSCLC tumors were used in the study. All the specimens were received as discarded materials from The Fourth Hospital of Jinan (Jinan, China) between March 2012 and March 2014. Lung cancers were divided into the following subtypes: Adenocarcinoma, squamous cell carcinoma, alveolar cell carcinoma and large cell carcinoma.

Immunohistochemistry (IHC). Formalin-fixed paraffin-embedded tissue sections (6- $\mu \mathrm{m}$ thick) were deparaffinized with xylene, rehydrated in descending concentrations of ethanol and boiled for $10 \mathrm{~min}$ in citrate buffer $(0.01 \mathrm{M}$, $\mathrm{pH}$ 6.0). Endogenous peroxidase activity was suppressed with $3 \% \mathrm{H}_{2} \mathrm{O}_{2}$ for $10 \mathrm{~min}$. Slides were serum-blocked (normal goat serum; Santa Cruz Biotechnology, Inc., Dallas, TX, USA) and incubated with primary antibodies against APRIL (1:200; ab64967; Abcam, Cambridge, UK), BCMA (1:200; ab5972; Abcam) and TACI (1:200; ab64967; Abcam) for $1 \mathrm{~h}$ at room temperature, and then stained with ImmunoCruz ${ }^{\mathrm{TM}}$ goat $\mathrm{ABC}$ Staining System (sc-2023; Santa Cruz Biotechnology, Inc.) according to the manufacturer's protocol. Counterstaining was performed using Harris hematoxylin. For negative controls, all the conditions were the same with the exception of the primary antibody, which was eliminated.

Cell culture and transfection. The human lung adenocarcinoma cell A549 line was purchased from the Type Culture 
Collection of the Chinese Academy of Sciences (Shanghai, China). The cells were maintained in Dulbecco's modified Eagle's medium (Gibco; Thermo Fisher Scientific, Inc., Waltham, MA, USA) supplemented with $10 \%$ fetal bovine serum (Invitrogen; Thermo Fisher Scientific, Inc.), 100 IU/ml penicillin and $100 \mu \mathrm{g} / \mathrm{ml}$ streptomycin at $37^{\circ} \mathrm{C}$ in a humidified $5 \% \mathrm{CO}_{2}$ atmosphere.

RNA isolation and reverse transcription-quantitative polymerase chain reaction $(R T-q P C R)$. Total RNAs were extracted with TRNzol-A+ Reagent (Tiangen Biotech Co., Ltd., Beijing, China) according to the manufacturer's protocol. RT-qPCR analysis of APRIL, BCMA and TACI was performed with $1 \mu \mathrm{g}$ of total RNA and RevertAid First Strand cDNA Synthesis kit (Fermentas; Thermo Fisher Scientific, Inc.). The primer sequences used were as follows: APRIL forward primer, 5'-TTC CCA TTA ACG CCA CCT CC-3' and reverse primer, 5'-CAG CAT CC TGG ATT CGG ACA-3'; BCMA forward primer, 5'-TGT TCT TCT AAT ACT CCT CCT CT-3' and reverse primer, 5'-AAC TCG TCC TTT AAT GGT TC-3'; and TACI forward primer, 5'-CCA TCT GTG GAC AGC ACC CT-3' and reverse primer, 5'-TTC CCG AGT TGT CTG AAT TGT TT-3'. Primers specific for $\beta$-actin were used as a control (forward, 5'-TCC ATC ATG AAG TGT GAC GT-3' and reverse, 5'-GAG CAA TGATCTTGATCT TCA T-3'). RT-qPCR was performed in a LightCycler $^{\circledR} 2.0$ Instrument (Roche Diagnostics, Basel, Switzerland) using LightCycler ${ }^{\circledR} 480$ SYBR Green I Master (Roche Diagnostics). PCR conditions consisted of the following: $95^{\circ} \mathrm{C}$ for $3 \mathrm{~min}$ for denaturation; $95^{\circ} \mathrm{C}$ for $30 \mathrm{sec}$ for annealing; and $62^{\circ} \mathrm{C}$ for $40 \mathrm{sec}$ for extension, for 40 cycles. The threshold cycle for each sample was selected from the linear range and converted to a starting quantity by interpolation from a standard curve generated on the same plate for each set of primers. The APRIL, BCMA and TACI messenger (m) RNA levels were normalized for each well to the $\beta$-actin mRNA levels using the $2^{-\Delta \Delta C q}$ method (12). Each experiment was repeated three times.

Immunoblot analysis. Tissue and whole-cell lysates were prepared with radioimmunoprecipitation assay (RIPA) buffer [10 mM Tris (pH 7.4), $150 \mathrm{mM} \mathrm{NaCl}, 1 \%$ Triton X-100, $0.1 \%$ deoxycholate, $0.1 \%$ sodium dodecyl sulfate (SDS) and $5 \mathrm{mM}$ ethylenediaminetetraacetic acid], containing $1 \mathrm{X}$ cOmplete ${ }^{\mathrm{TM}}$ protease inhibitor cocktail (Roche Diagnostics). Lysates were normalized for total protein $(25 \mu \mathrm{g})$ and subjected to $10 \%$ SDS-polyacrylamide gel electrophoresis (PAGE) and immunoblot assay. The proteins were transferred to a polyvinylidene difluoride membrane (EMD Millipore, Billerica, MA, USA), followed by blocking with $5 \%$ skimmed milk at room temperature for $1 \mathrm{~h}$. The membrane was then incubated with primary antibodies overnight at $4{ }^{\circ} \mathrm{C}$ and rinsed with Tris-buffered saline with Tween 20 . The following primary antibodies were used: Phospho-ERK1/2 (1:1,000 dilution; catalog no. 9101; Cell Signaling Technology, Inc., Danvers, MA, USA), APRIL (1:1,000 dilution; catalog no. ab64967; Abcam), BCMA (1:1,000 dilution; catalog no. ab5972; Abcam) and TACI (1:1,000 dilution; catalog no. ab64967; Abcam). The blots were then incubated with goat anti-rabbit immunoglobulin G H\&L horseradish peroxidase (HRP)-conjugated secondary antibodies (catalog no. ab6721; Abcam) prior to being subjected to enhanced chemiluminescence detection (Luminata Western HRP Substrate; EMD Millipore) and subsequent autoradiography with Super RX film (Fujifilm, Tokyo, Japan). The defined sections of the film were scanned for density measurement and analyzed using ImageJ software (National Institutes of Health, Bethesda, MD, USA).

Statistical analysis. Experimental data were presented as the mean \pm standard error of the mean. All statistical analyses were performed with use of the SPSS 13.5 (SPSS, Inc., Chicago, IL, USA). Student's $t$ test and one-way analysis of variance, followed by Tukey's post hoc analysis, was applied. $\mathrm{P}<0.05$ was considered to indicate a statistically significant difference.

\section{Results}

IHC detection of APRIL in NSCLC specimens. Previous reports established that APRIL, BCMA and TACI are expressed in various tumors, but not in normal tissues $(10,13)$. The present study assessed their expression in 38 resected paraffin-embedded NSCLC tumor samples by IHC. Among the tumor specimens examined, 38 cases of NSCLC and 9 cases of normal lung tissue in the neighborhood of the main lesion were identified. APRIL was positive in 27/38 cases of cancer and in 1/9 normal lung tissues. Typical cases are presented in Fig. 1. APRIL immunoreactivity was detected in the cytoplasm. These results indicate that elevated APRIL levels are common in NSCLC, thus suggesting a functional role for APRIL in lung cancers.

IHC detection of BCMA and TACI in NSCLC specimens. The present results revealed that BCMA and TACI are overexpressed in NSCLC samples. BCMA was positive in $28 / 38$ cases of cancer and in 3/9 normal lung tissues, while TACI was positive in 29/38 cases of cancer and in 2/9 normal lung tissues. Typical cases are presented in Fig. 2. BCMA and TACI immunoreactivity was detected in the cytoplasm and in the cell surface.

APRIL, BCMA and TACI are overexpressed in NSCLC specimens, as detected by WB and RT-qPCR. To further confirm the results of IHC, APRIL, BCMA and TACI levels were measured by qPCR in a panel of NSCLC samples containing normal tissue. As indicated in Fig. 3A, APRIL transcripts were detected in the majority of cancer samples (15/18), with significantly increased levels compared with normal tissues $(\mathrm{P}=0.032)$. For the receptors, a greater incidence of BCMA and TACI transcripts was observed in cancer samples than in normal samples (Fig. 3). Similar to the RT-qPCR results, APRIL, BCMA and TACI protein levels were significantly higher in cancer samples than in normal tissues by WB, suggesting a direct correlation between the APRIL/BCMA/TACI pathway and NSCLC development.

APRIL activates the MAPK ERK1/2 in A549 cells. The finding that NSCLC overexpresses APRIL, TACI and BCMA predicted that signaling through these receptors may promote tumor growth. Therefore, the present study analyzed 

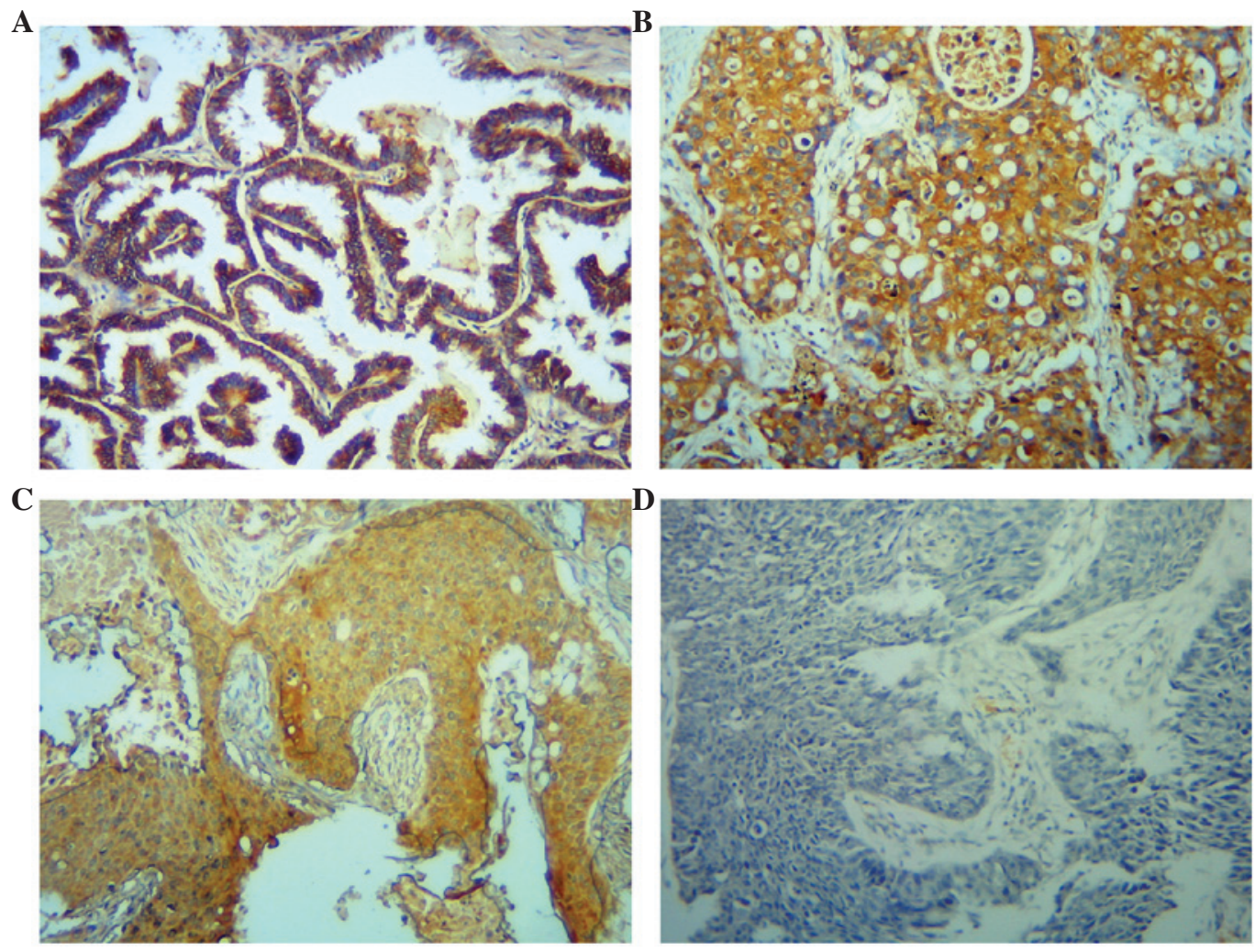

Figure 1. APRIL expression is increased in NSCLC samples. (A-C) Immunohistochemistry study of APRIL expression in paraffin-embedded NSCLC samples. Representative images of APRIL staining (brown) in (A) adenocarcinoma and (B and C) squamous cell carcinoma. (D) Representative images of negative staining (without primary antibody) of the sample in panel C. Magnification, x100. APRIL, a proliferation-inducing ligand; NSCLC, non-small cell lung cancer.
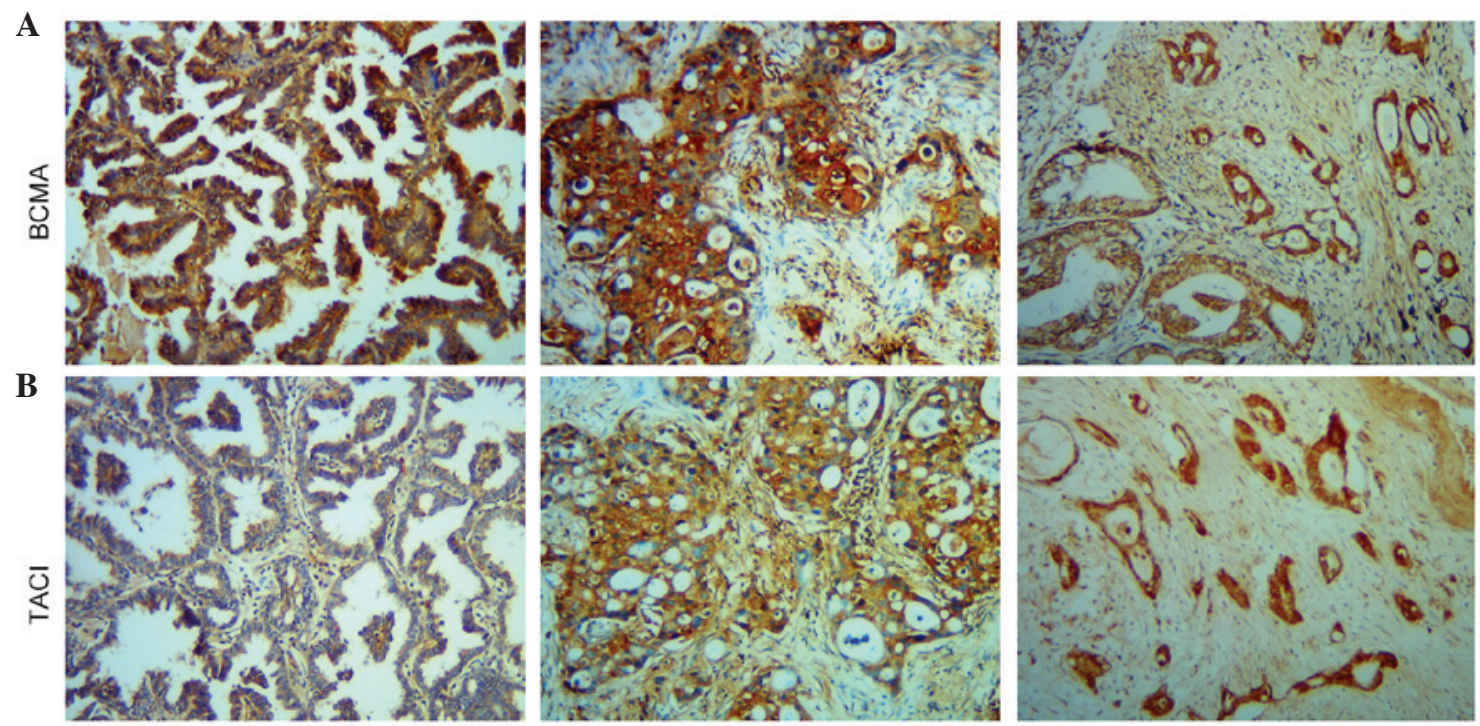

Figure 2. BCMA and TACI expression is increased in NSCLC samples. Immunohistochemistry study of BCMA and TACI expression in paraffin-embedded NSCLC samples. (A) Representative images of BCMA staining (brown). (B) Representative images of TACI staining (brown) in lung cancer. Magnification, $\mathrm{x} 100$. BCMA, B-cell maturation antigen; TACI, transmembrane activator and CAML interactor; NSCLC, non-small cell lung cancer.

whether APRIL mediated signaling via the MAPK ERK1/2, which has been implicated in NSCLC tumorigenesis (14-18). Analysis of phosphorylation kinetics in A549 cells revealed that APRIL overexpression induced the phosphorylation of ERK1/2 (Fig. 4A). In addition, overexpression of BCMA and TACI was observed. The enhanced receptor expression may also improve the ability of cells to respond to APRIL.
To assess this hypothesis, BCMA and TACI expression was reduced in A549 cells using small interference (si)RNA, and the ERK1/2 phosphorylation level upon APRIL overexpression was detected. The results confirmed that BCMA and TACI knockdown could reduce the ERK1/2 phosphorylation level in both basal conditions and in the APRIL-overexpressing group. 
A

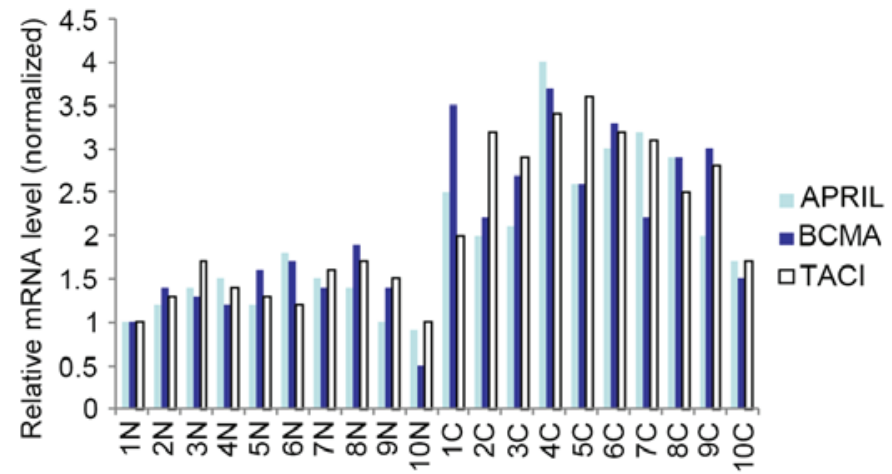

B

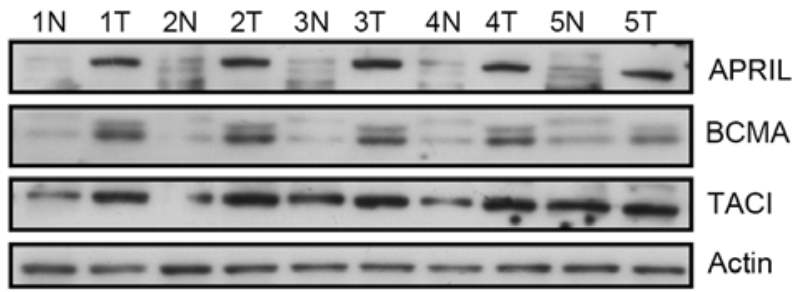

Figure 3. APRIL, BCMA and TACI expression profile in NSCLC tissue samples, as analyzed by WB and RT-qPCR. (A) mRNA expression of APRIL, BCMA and TACI in NSCLC samples, as measured by RT-qPCR, relative to that of $\beta$-actin. (B) Protein expression of BCMA and TACI in NSCLC samples, as measured by WB, relative to that of $\beta$-actin. APRIL, a proliferation-inducing ligand; BCMA, B-cell maturation antigen; TACI, transmembrane activator and CAML interactor; NSCLC, non-small cell lung cancer; N, normal; T, tumor; C, cancer; mRNA, messenger RNA; RT-qPCR, reverse transcription-quantitative polymerase chain reaction; WB, western blotting.

A

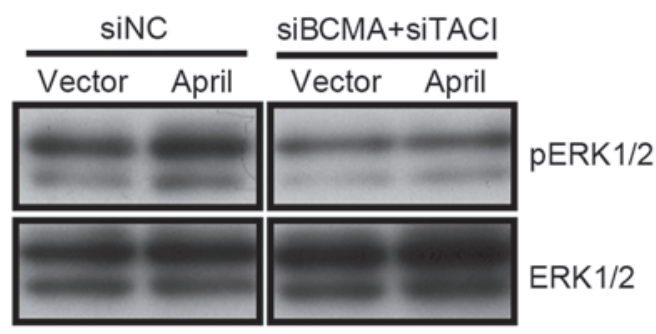

B

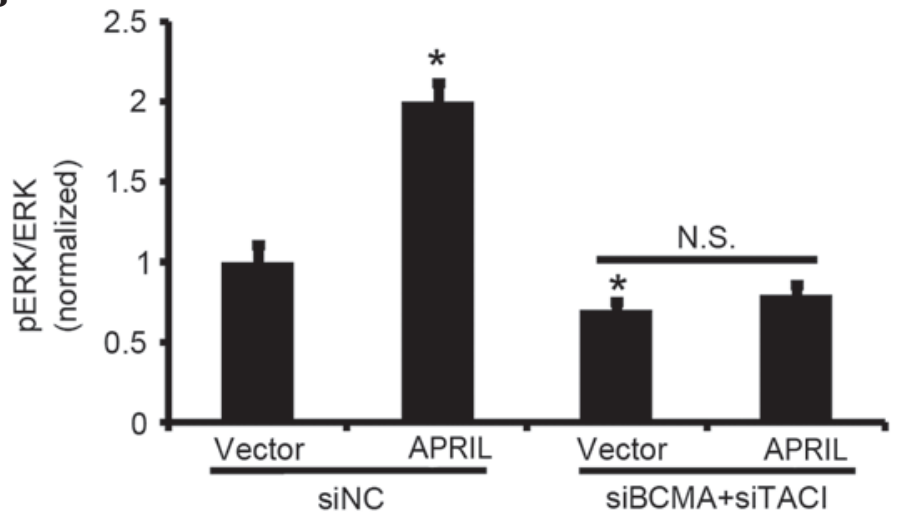

Figure 4. APRIL activates the mitogen-activated protein kinase ERK1/2 in A549 cells. (A) Extracts from A549 cells transfected with BCMA siRNA and TACI siRNA were subjected to western blotting and probed with antibodies against pERK1/2 and total ERK1/2. (B) Bar graphs represent the mean \pm SEM ( $\mathrm{n}=5$; "P<0.05, one-way ANOVA). si, small interfering; NC, negative control; N.S., not significant; APRIL, a proliferation-inducing ligand; BCMA, B-cell maturation antigen; TACI, transmembrane activator and CAML interactor; ERK, extracellular signal regulated kinase; p, phosphorylated; WB, western blotting; ANOVA, analysis of variance; SEM, standard error of the mean.si, small interfering; NC, negative control; N.S., not significant; APRIL, a proliferation-inducing ligand; BCMA, B-cell maturation antigen; TACI, transmembrane activator and CAML interactor; ERK, extracellular signal-regulated kinase; p, phosphorylated; WB, western blotting; ANOVA, analysis of variance; SEM, standard error of the mean.

\section{Discussion}

APRIL was initially described in the 1990s based on its ability to induce the proliferation of distinct types of cell lines (9). The initial data demonstrated that APRIL is overexpressed in leukemia and lymphoma, and promotes tumorigenesis, while later reports indicated a role for this cytokine beyond hematopoietic malignancies $(11,19-24)$.

There are currently no data regarding APRIL, BCMA and TACI expression in NSCLC. The present study detected expression of all three proteins in 38 primary lung carcinomas and in one lung cancer cell line. Higher levels of APRIL,
BCMA and TACI protein and mRNA transcripts in NSCLC than in normal tissues were detected, indicating that the overexpression of APRIL, BCMA and TACI in human NSCLC serves a role in carcinogenesis and results in a poorer clinical outcome.

Co-expression of APRIL and its receptors enable autocrine proliferation of tumor cells, as confirmed in APRIL overexpression assays. It was demonstrated that APRIL expression induces the phosphorylation of ERK1/2 in A549 cells. MAPKs are overexpressed in NSCLC, and their activation enhances cancer cell survival and proliferation (16-18). Switching on the APRIL pathway may provide malignant 
cells with a mechanism to sustain activation of their MAPK signaling and promote tumor progression. The present study demonstrated that NSCLC expressed BCMA and TACI; thus, it is possible that both receptors enhance the cells' response to APRIL. In addition, BCMA and TACI expression was reduced in A549 cells using siRNA, and the results confirmed that the expression levels of BCMA and TACI are associated with APRIL-dependent ERK1/2 activation.

In summary, the current study reports the overexpression of APRIL, BCMA and TACI in NSCLC samples and cell lines, and has identified an APRIL signaling pathway that functions and participates in human lung tumorigenesis.

\section{Acknowledgements}

The present study was funded by a grant from the Science and Technology Program of Jinan, China (no. 201101195).

\section{References}

1. Detterbeck FC, Postmus PE and Tanoue LT: The stage classification of lung cancer: Diagnosis and management of lung cancer, 3rd edition. American college of chest physicians evidence-based clinical practice guidelines. Chest 143 (Suppl 5): e191S-e210S, 2013.

2. Chen Z, Xu Z, Sun S, Yu Y, Lv D, Cao C and Deng Z: TGF- $\beta 1$, IL- 6 and TNF- $\alpha$ in bronchoalveolar lavage fluid: Useful markers for lung cancer? Sci Rep 4: 5595, 2014.

3. Chyczewska E, Mróz RM and Kowal E: TNF-alpha, IL-1 and IL-6 concentration in bronchoalveolar lavage fluid (BALF) of non-small cell lung cancer (NSCLC). Rocz Akad Med Bialymst 42 (Suppl 1): S123-S135, 1997.

4. Liu H, Zhang T, Ye J, Li H, Huang J, Li X, Wu B, Huang X and Hou J: TNF receptor-associated factor 6 in advanced non-small cell lung cancer: Clinical and prognostic implications. J Cancer Res Clin Oncol 138: 1853-1863, 2012.

5. O'Connell C, Hensey M, Mongey AB, Veale DJ and Donnelly SC: A series of patients on anti-TNF therapy referred to a multidisciplinary lung cancer service. Ir J Med Sci 182: 135-137, 2013.

6. Seifart C, Plagens A, Dempfle A, Clostermann U, Vogelmeier C, von Wichert $\mathrm{P}$ and Seifart U: TNF-alpha, TNF-beta, IL-6 and IL-10 polymorphisms in patients with lung cancer. Dis Markers 21: 157-165, 2005.

7. Xie H, Yao H, Huo Y, Li N and Cheng Y: Association between TNF- $\alpha$ gene $308 \mathrm{G}>\mathrm{A}$ polymorphism and lung cancer risk: A meta-analysis. Tumour Biol 35: 9693-9699, 2014.

8. Zhang B and Wu Y: The preoperative and postoperative changes of T lymphocyte subsets and TNF in patients with locally advanced lung cancer. Zhongguo Fei Ai Za Zhi 2: 67-69, 1999 (In Chinese).

9. Hahne M, Kataoka T, Schröter M, Hofmann K, Irmler M, Bodmer JL, Schneider P, Bornand T, Holler N, French LE, et al: APRIL, a new ligand of the tumor necrosis factor family, stimulates tumor cell growth. J Exp Med 188: 1185-1190, 1998.

10. Moreaux J, Veyrune JL, De Vos J and Klein B: APRIL is overexpressed in cancer: Link with tumor progression. BMC Cancer 9: $83,2009$.
11. Notas G, Alexaki VI, Kampa M, Pelekanou V, Charalampopoulos I, Sabour-Alaoui S, Pediaditakis I, Dessirier V, Gravanis A, Stathopoulos EN, et al: APRIL binding to BCMA activates a JNK2-FOXO3-GADD45 pathway and induces a G2/M cell growth arrest in liver cells. J Immunol 189: 4748-4758, 2012.

12. Livak and Schmittgen: Analysis of relative gene expression data using real-time quantitative PCR and the $2-\Delta \Delta \mathrm{Ct}$ method. Methods 25: 402-408, 2001.

13. Garcia-Castro A, Zonca M, Florindo-Pinheiro D, Carvalho-Pinto CE, Cordero A, Gutiérrez del Fernando B, García-Grande A, Mañes S, Hahne M, González-Suárez E and Planelles L: APRIL promotes breast tumor growth and metastasis and is associated with aggressive basal breast cancer. Carcinogenesis 36: 574-584, 2015.

14. Kim CH, Han SI, Lee SY, Youk HS, Moon JY, Duong HQ, Park MJ, Joo YM, Park HG, Kim YJ, et al: Protein kinase C-ERK1/2 signal pathway switches glucose depletion-induced necrosis to apoptosis by regulating superoxide dismutases and suppressing reactive oxygen species production in A549 lung cancer cells. J Cell Physiol 211: 371-385, 2007.

15. Ko JC, Ciou SC, Jhan JY, Cheng CM, Su YJ, Chuang SM, Lin ST, Chang CC and Lin YW: Roles of MKK1/2-ERK1/2 and phosphoinositide 3-kinase-AKT signaling pathways in erlotinib-induced Rad51 suppression and cytotoxicity in human non-small cell lung cancer cells. Mol Cancer Res 7: 1378-1389, 2009.

16. Vicent S, Lopez-Picazo JM, Toledo G, Lozano MD, Torre W, Garcia-Corchón C, Quero C, Soria JC, Martín-Algarra S, Manzano RG and Montuenga LM: ERK1/2 is activated in non-small-cell lung cancer and associated with advanced tumours. Br J Cancer 90: 1047-1052, 2004.

17. Wang $\mathrm{H}$, Wu C, Wan S, Zhang H, Zhou S and Liu G: Shikonin attenuates lung cancer cell adhesion to extracellular matrix and metastasis by inhibiting integrin $\beta 1$ expression and the ERK1/2 signaling pathway. Toxicology 308: 104-112, 2013.

18. Wang M, Liu ZM, Li XC, Yao YT and Yin ZX: Activation of ERK1/2 and Akt is associated with cisplatin resistance in human lung cancer cells. J Chemother 25: 162-169, 2013.

19. Mhawech-Fauceglia P, Kaya G, Sauter G, McKee T, Donze O, Schwaller J and Huard B: The source of APRIL up-regulation in human solid tumor lesions. J Leukoc Biol 80: 697-704, 2006.

20. Mhawech-Fauceglia P, Allal A, Odunsi K, Andrews C, Herrmann FR and Huard B: Role of the tumour necrosis family ligand APRIL in solid tumour development: Retrospective studies in bladder, ovarian and head and neck carcinomas. Eur J Cancer 44: 2097-2100, 2008.

21. Pelekanou V, Notas G, Theodoropoulou K, Kampa M, Takos D, Alexaki VI, Radojicic J, Sofras F, Tsapis A, Stathopoulos EN and Castanas E: Detection of the TNFSF members BAFF, APRIL, TWEAK and their receptors in normal kidney and renal cell carcinomas. Anal Cell Pathol (Amst) 34: 49-60, 2011.

22. Pelekanou V, Notas G, Kampa M, Tsentelierou E, Stathopoulos EN, Tsapis A and Castanas E: BAFF, APRIL, TWEAK, BCMA, TACI and Fn14 proteins are related to human glioma tumor grade: Immunohistochemistry and public microarray data meta-analysis. PloS One 8: e83250, 2013.

23. Wang F, Chen L, Mao ZB, Shao JG, Tan C and Huang WD: Lentivirus-mediated short hairpin RNA targeting the APRIL gene suppresses the growth of pancreatic cancer cells in vitro and in vivo. Oncol Rep 20: 135-139, 2008.

24. Lascano V, Zabalegui LF, Cameron K, Guadagnoli M, Jansen M, Burggraaf M, Versloot M, Rodermond $\mathrm{H}$, van der Loos C, Carvalho-Pinto CE, et al: The TNF family member APRIL promotes colorectal tumorigenesis. Cell Death Differ 19: 1826-1835, 2012. 